\title{
Effects of a Facial Cream Containing the Minor Alkaloid Anatabine on Improving the Appearance of the Skin in Mild to Moderate Rosacea: An Open-Label Case Series Study
}

\author{
Ryan K. Lanier $^{a} \quad$ Amy E. Cohen $^{a} \quad$ Susan H. Weinkle \\ ${ }^{a}$ Rock Creek Pharmaceuticals, Inc., Gloucester, Mass., and ${ }^{b}$ Private practice, Bradenton, \\ Fla., USA
}

\section{Key Words}

Anatabine $\cdot$ Rosacea $\cdot$ Inflammation $\cdot$ Erythema $\cdot$ Facial redness $\cdot$ Topical

\begin{abstract}
Background: Current medical and scientific research indicates that rosacea, a chronic and often debilitating skin condition that primarily affects the central face, may be caused by an overactive or excessive inflammatory immune response. Regardless of etiology, the accompanying redness and inflammation is unsightly and difficult for the patient. Anatabine is an alkaloid from the plant family Solanaceae that has been shown in several preclinical studies to modulate proinflammatory signaling pathways. Objective: A cream containing anatabine was developed and evaluated in an open-label case series study for safety and effects on the appearance of the skin in 10 patients with mild to moderate rosacea. Methods: Patients applied the cream to the face twice daily for a period of 30 days. Patients and the study physician completed safety and efficacy assessments at study end. Results: Results showed that $50 \%$ of the patients self-reported improvement in the appearance of their skin, and the physician noted improvement in $70 \%$ of the patients. Photographs taken before and after 30 days of cream use provide visual evidence of the improvement in several patients. There were no complications or adverse events reported by any of the patients in the study, indicating that the anatabine cream was safe and very well tolerated. Conclusion: The results
\end{abstract}


of this open-label case series show that a facial cream containing anatabine can improve the appearance of the skin in patients with mild to moderate rosacea and suggest that a doubleblind, vehicle-controlled trial in a larger number of subjects is warranted.

(c) 2013 S. Karger AG, Basel

\section{Introduction}

Rosacea is a common, progressive, chronic, and in many cases cyclic, inflammatory skin disorder that presents with a variety of clinical manifestations that are primarily localized to the central face. Rosacea affects up to $10 \%$ of the US population and occurs most commonly in adults between 30 and 60 years of age and in fair-skinned individuals of Celtic and northern European heritage [1, 2]. The disorder is divided into four main subtypes based upon groupings of primary and secondary features: (1) erythematotelangiectatic (centrofacial redness); (2) papulopustular (acne-like inflammatory papules and pustules); (3) phymatous (marked skin thickening and distortion of facial contours, more often in men), and (4) ocular rosacea (eye redness, pruritus, or irritation). Granulomatous rosacea is a variant of rosacea (yellow-brown or pink papules) [3].

The cause of rosacea is unclear, and many hypotheses have been proposed based upon the numerous contributing factors and triggers that lead to flare ups of rosacea, including sun exposure, extreme temperature, spicy food, alcohol, stress, topical irritation, and drugs (vasodilators, nicotinic acid). Multiple lines of evidence in recent years have led many experts to believe that rosacea is primarily caused and exacerbated by an overactive inflammatory immune response. It has been hypothesized that excessive upregulation of inflammatory mediators and/or dysregulation of innate immune function may underlie the diverse range of rosacea signs and symptoms including vasodilation, redness, angiogenesis, edema, and the formation of papules and pustules [4-6]. In this model, environmental stressors (ultraviolet light radiation, heat, cold, spicy food, and alcohol), microbes, and certain types of bacteria stimulate an innate immune system signaling cascade leading to the overproduction and activation of cytokines, chemokines, antimicrobial peptides (cathelicidins), and other proinflammatory mediators that result in tissue destruction and infiltration by inflammatory cells such as neutrophils and lymphocytes. This excessive, uncontrolled inflammatory response is believed to be responsible for the clinical manifestations of rosacea, and many researchers suggest that targeting and controlling excess inflammation should be the goal for new rosacea therapeutics in development [4, 6-8]. Regardless of the etiology, the consequent redness and inflammation of the face in rosacea is difficult for the patient, does not respond particularly well to treatment, and is frustrating for both physician and patient.

Topical and oral medications used to treat rosacea have equivocal clinical efficacy, and there is a recognized need for more effective, safe, and well-tolerated long-term treatment options. Compounds that alleviate or control excessive inflammation in the skin are likely to provide relief from many of the symptoms of rosacea, and many rosacea sufferers are beginning to experiment with new, non-prescription treatments with unproven safety or efficacy. Briefly put, there are no current effective ways for a patient with rosacea to reliably improve the appearance of their skin.

Anatabine is a minor alkaloid found in plants of the Solanaceae family, including peppers, tomatoes, potatoes, eggplant, and tobacco, that is being investigated as a modulator of systemic inflammation. Researchers have shown that anatabine exhibits anti-inflammatory effects in a number of in vivo and in vitro studies. In a transgenic model of Alzheimer's 
disease, anatabine administration resulted in the downregulation of the proinflammatory molecule amyloid $\beta$ due to reduced activation of nuclear factor $\kappa \mathrm{B}$ (NF- $\kappa \mathrm{B})$-dependent pathways, and anatabine significantly lowered plasma levels of the inflammatory marker Creactive protein in these animals [9]. Anatabine has also been shown to block the production of proinflammatory cytokines such as IL-1 $\beta$, IL-6, and TNF- $\alpha$ in the blood and tissues of mice injected with lipopolysaccharide by blocking the activation of the transcription factors NF$\kappa \mathrm{B}$ and STAT3 [10]. Caturegli et al. [11] investigated the effects of anatabine in a mouse model of autoimmune thyroiditis and found that anatabine significantly reduced several markers of systemic inflammation.

Although the precise mechanism underlying the diverse immunomodulatory effects of anatabine is unclear, a topical cream containing anatabine may provide anti-inflammatory benefits when applied to the skin by interfering with proinflammatory signaling pathways. The purpose of this open-label case series study was to examine the safety, tolerability, and effects of a facial cream containing anatabine in patients with mild to moderate rosacea, principally to determine if it could improve the appearance of the skin.

\section{Methods}

This case series was conducted at a private dermatology clinic located in Bradenton, Fla., USA. Patients interested in taking part in this case series provided written informed consent, which included consent to use facial photographs for scientific presentation or publication, before any study-related procedures took place. This study did not require ethics approval. Each patient was treated as clinically indicated, and patient confidentiality was protected. On day 1, patients came to the clinic for baseline assessment, had photographs taken, and received instructions for using the anatabine cream. For the duration of the 30-day study period, patients were instructed to use the anatabine cream twice daily. Patients rated 6 components of their baseline skin appearance and rosacea symptoms (overall skin appearance; appearance of rosacea; redness/blotchiness; skin tone and color; blemishes, and dryness/flakiness) on a 4-point scale from 0 (absent) to +3 (severe) by filling out the Patient Global Assessment (PatGA), and the physician rated the cardinal signs of rosacea using the Physician's Global Assessment (PhyGA), as described by Wilkin et al. [12]. Clinic staff called patients approximately 2 weeks after day 1 to ask about their experience using the cream. Patients returned to the clinic approximately 30 days after day 1 for final assessments and photographs. At this visit, patients and the physician rated the effectiveness of the cream by filling out the Patient Efficacy Assessment (PatEA) and the Physician's Efficacy Assessment (PhyEA), respectively. The PatEA and PhyEA each consisted of a 5-point rating scale from -1 (worse) to +3 (cleared up). All patients were questioned for any adverse events, side effects, or complaints they experienced during the 30-day treatment period.

\section{Results}

A total of 10 acne rosacea patients ( 7 women, 3 men; mean age $69.0 \pm 8.58$ years; age range 51-81 years; all Caucasian) took part in this case series. At baseline, all study patients self-reported the overall appearance of rosacea to be either mild (1 of 10,10\%) or moderate ( 9 of $10,90 \%$ ) on the PatGA. Redness and blotchiness were reported as mild for 5 (50\%) of the patients and moderate for the remaining $5(50 \%)$ patients (fig. 1). The physician assessed that all of the patients had either subtype 1 (erythematotelangiectatic) or subtype 2 
(papulopustular) rosacea, or a combination of the two (table 1 ). The majority of patients (8 of $10,80 \%$ ) had moderate subtype 1 rosacea. Other results from the PhyGA indicated that, for all patients, flushing was mild ( 4 of $10,40 \%$ ) or moderate ( 6 of $10,60 \%$ ); erythema was mild ( 2 of $10,20 \%$ ) or moderate ( 8 of $10,80 \%)$; papules and pustules were absent ( 4 of 10 , $40 \%)$, mild ( 3 of $10,30 \%)$, or moderate ( 3 of $10,30 \%)$, and telangiectasia was moderate $(9$ of $10,90 \%)$ or severe $(1$ of $10,10 \%)$. Secondary features of rosacea were noted to be absent in these patients.

At the end of the 30-day period, the physician assessed the effectiveness of the cream using the PhyEA. Rosacea improvement was noted in 7 of the $10(70 \%)$ patients; no improvement was observed in $2(20 \%)$ patients, and worsening occurred in $1(10 \%)$ patient (fig. 2). The study patients self-assessed their rosacea using the PatEA. Five (50\%) patients reported rosacea improvement, 4 (40\%) patients reported no improvement, and $1(10 \%)$ patient reported worsening of their skin (fig. 2). Representative before and after photographs showing marked improvement in overall rosacea appearance for 2 patients following 30 days of anatabine cream use are shown in figure 3.

\section{Discussion}

In the present open-label case series study, twice daily application of a topical facial cream containing the minor alkaloid anatabine was shown to improve the appearance of the skin in patients with mild to moderate rosacea. As rated by the physician, skin appearance improved in $70 \%$ of the patients, and $50 \%$ of the patients self-reported an improvement following 30 days of topical application. There were no complications or adverse events reported by any of the patients in the case series, indicating that the investigative product was safe and very well tolerated.

Anatabine has been shown in multiple in vitro and in vivo models of excessive inflammation to attenuate the production of proinflammatory mediators through a reduction in the activation of the transcription factors NF- $\mathrm{KB}$ and STAT3 $[9-11,13]$. Research has shown that $\mathrm{NF}-\kappa \mathrm{B}$ is involved in the upregulation of the antimicrobial peptides (cathelicidins) and other proinflammatory mediators believed to be involved in the progression and severity of rosacea $[14,15]$. Mastrofrancesco et al. [16] showed that the topical rosacea treatment azelaic acid inhibited ultraviolet light-induced activation and translocation of NF- $\mathrm{KB}$ and reduced the production of proinflammatory cytokines in human keratinocytes. Therefore, anatabine may provide anti-inflammatory benefits when applied topically by interfering with NF- $\kappa B$ activation, or through inhibition of some other related proinflammatory signaling mechanism (fig. 4).

An important consideration when evaluating a topically applied product containing a plant-based compound for a condition characterized by sensitive skin such as rosacea is its risk of inducing contact dermatitis $[17,18]$. Anatabine is an alkaloid found in a wide range of Solanaceae family plants, including many commonly consumed food staples such as tomatoes and potatoes, but to date, there have been no published reports of any hypersensitivity or allergic reactions directly attributed to the ingestion of or skin exposure to anatabine. Results from this case series suggest that contact dermatitis was not an issue for the patients using the anatabine cream; however, future studies of the cream in larger populations of patients should further evaluate this possibility.

Most patients in this case series had moderate erythematotelangiectatic rosacea, which is characterized by persistent flushing and redness, and may include stinging, burning, and swelling. These particular signs and symptoms of rosacea could be due to excessive, 
uncontrolled inflammation in the skin, and thus may respond particularly well to treatment with a topical anatabine preparation. It is uncertain if other subtypes of rosacea, particularly those involving papules and pustules and/or tissue/vascular damage, would respond as favorably. Further studies focusing on the effects of an anatabine-based cream on other subtypes of rosacea would provide more insight into the actions of this compound. It must be noted that interpretation of the results of this case series is limited due to the small sample size and lack of a control group.

The primary objectives of this case series were to determine if twice daily application of the anatabine cream was safe and if it would improve the appearance of the skin in a small sample of patients primarily suffering from persistent erythema due to rosacea. Both objectives were met, although there was some disparity in the perceived effectiveness of the cream between the patients and the physician, as indicated by the PatEA and the PhyEA assessment scores. The physician rated a greater degree of improvement (i.e. 1 or 2 points) than the patient in 6 of 10 cases, whereas only 1 patient's self-reported rating of improvement was greater than the physician's rating. This difference in perceived outcome could be due to the fact that patients saw themselves every day and might not have noticed changes happening over time, whereas the physician saw them once at baseline, and then again after 30 days. Other studies of rosacea treatments have resulted in a similar phenomenon in which investigator ratings of success or improvement were typically of a greater magnitude or frequency than study subjects' ratings, although not necessarily statistically different [1922]. A possible solution to reduce or eliminate this type of rating disparity between observer and self-reported measures would be the use of before and after photographs for comparison purposes for both physician and patient use.

In conclusion, results from this open-label case series show that daily application of a topical facial cream containing the minor alkaloid anatabine is safe and well tolerated and can reduce the signs and symptoms of mild to moderate rosacea. Improving the appearance of the skin by reducing persistent erythema, and possibly other signs and symptoms of rosacea, could lead to an overall increase in the quality of life for those dealing with this chronic disease. A larger, double-blind, vehicle-controlled trial of the anatabine facial cream in subjects with mild to moderate rosacea appears to be warranted.

\section{Acknowledgements}

The authors would like to thank Theresa Hausman for her assistance and coordinating role in this study, and Jane Loescher for her assistance with data management and manuscript preparation.

\section{Disclosure Statement}

The anatabine cream was supplied by Rock Creek Pharmaceuticals (RCP), Inc. R.K.L. and A.E.C. are employees of RCP. S.H.W. received no funding for this study from RCP, and has no conflicts of interest to declare. 
Lanier et al.: Effects of a Facial Cream Containing the Minor Alkaloid Anatabine on Improving the Appearance of the Skin in Mild to Moderate Rosacea

\section{References}

1 Powell FC: Clinical practice. Rosacea. N Engl J Med 2005;352:793-803.

2 van Zuuren EJ, Kramer S, Carter B, Graber MA, Fedorowicz Z: Interventions for rosacea. Cochrane Database Syst Rev 2011;3:CD003262.

-3 Wilkin J, Dahl M, Detmar M, Drake L, Feinstein A, Odom R, Powell F: Standard classification of rosacea: report of the National Rosacea Society Expert Committee on the classification and staging of rosacea. J Am Acad Dermatol 2002;46:584-587.

4 Yamasaki K, Gallo RL: The molecular pathology of rosacea. J Dermatol Sci 2009;55:77-81.

5 Gerber PA, Buhren BA, Steinhoff M, Homey B: Rosacea: the cytokine and chemokine network. J Investig Dermatol Symp Proc 2011;15:40-47.

-6 Del Rosso JQ, Gallo RL, Tanghetti E, Webster G, Thiboutot D: An evaluation of potential correlations between pathophysiologic mechanisms, clinical manifestations, and management of rosacea. Cutis 2013;91(3 suppl):1-8.

7 Yamasaki K, Gallo RL: Rosacea as a disease of cathelicidins and skin innate immunity. J Investig Dermatol Symp Proc 2011;15:12-15.

-8 Elewski BE, Draelos Z, Dréno B, Jansen T, Layton A, Picardo M: Rosacea - global diversity and optimized outcome: proposed international consensus from the Rosacea International Expert Group. J Eur Acad Dermatol Venereol 2011;25:188-200.

-9 Paris D, Beaulieu-Abdelahad D, Bachmeier C, Reed J, Ait-Ghezala G, Bishop A, Chao J, Mathura V, Crawford F, Mullan M: Anatabine lowers Alzheimer's Aß production in vitro and in vivo. Eur J Pharmacol 2011;670:384391.

10 Paris D, Beaulieu-Abdelahad D, Abdullah L, Bachmeier C, Ait-Ghezala G, Reed J, Verma M, Crawford F, Mullan M: Anti-inflammatory activity of anatabine via inhibition of STAT3 phosphorylation. Eur J Pharmacol 2013;698:145-153.

11 Caturegli P, De Remigis A, Ferlito M, Landek-Salgado MA, Iwama S, Tzou SC, Ladenson PW: Anatabine ameliorates experimental autoimmune thyroiditis. Endocrinology 2012;153:4580-4587.

12 Wilkin J, Dahl M, Detmar M, Drake L, Liang MH, Odom R, Powell F: Standard grading system for rosacea: report of the National Rosacea Society Expert Committee on the classification and staging of rosacea. J Am Acad Dermatol 2004;50:907-912.

13 Paris D, Beaulieu-Abdelahad D, Mullan M, Ait-Ghezala G, Mathura V, Bachmeier C, Crawford F, Mullan MJ: Amelioration of experimental autoimmune encephalomyelitis by anatabine. PLoS One 2013;8:e55392.

14 Wu H, Zhang G, Minton JE, Ross CR, Blecha F: Regulation of cathelicidin gene expression: induction by lipopolysaccharide, interleukin-6, retinoic acid, and Salmonella enterica serovar typhimurium infection. Infect Immun 2000;68:5552-5558.

15 Li G, Domenico J, Jia Y, Lucas JJ, Gelfand EW: NF-kappaB-dependent induction of cathelicidin-related antimicrobial peptide in murine mast cells by lipopolysaccharide. Int Arch Allergy Immunol 2009;150:122132.

16 Mastrofrancesco A, Ottaviani M, Aspite N, Cardinali G, Izzo E, Graupe K, Zouboulis CC, Camera E, Picardo M: Azelaic acid modulates the inflammatory response in normal human keratinocytes through PPARgamma activation. Exp Dermatol 2010;19:813-820.

17 Kiken DA, Cohen DE: Contact dermatitis to botanical extracts. Am J Contact Dermat 2002;13:148-152.

18 Thomson KF, Wilkinson SM: Allergic contact dermatitis to plant extracts in patients with cosmetic dermatitis. Br J Dermatol 2000;142:84-88.

$\checkmark 19$ Elewski BE, Fleischer AB Jr, Pariser DM: A comparison of $15 \%$ azelaic acid gel and $0.75 \%$ metronidazole gel in the topical treatment of papulopustular rosacea: results of a randomized trial. Arch Dermatol 2003;139:1444-1450.

20 Marks R, Ellis J: Comparative effectiveness of tetracycline and ampicillin in rosacea. A controlled trial. Lancet 1971;2:1049-1052.

21 Rigopoulos D, Kalogeromitros D, Gregoriou S, Pacouret JM, Koch C, Fisher N, Bachmann K, Brown M, Schwarz E, Camel E, Katsambas A: Randomized placebo-controlled trial of a flavonoid-rich plant extractbased cream in the treatment of rosacea. J Eur Acad Dermatol Venereol 2005;19:564-568.

22 Thiboutot D, Thieroff-Ekerdt R, Graupe K: Efficacy and safety of azelaic acid (15\%) gel as a new treatment for papulopustular rosacea: results from two vehicle-controlled, randomized phase III studies. J Am Acad Dermatol 2003;48:836-845. 
Lanier et al.: Effects of a Facial Cream Containing the Minor Alkaloid Anatabine on

Table 1. PhyGA of rosacea at baseline

Study participants $(\mathrm{n}=10)$

n (\%)

\begin{tabular}{|c|c|}
\hline \multicolumn{2}{|c|}{ Primary features of rosacea } \\
\hline $\begin{array}{l}\text { Flushing } \\
\text { Mild }\end{array}$ & $4(40)$ \\
\hline Moderate & $6(60)$ \\
\hline \multicolumn{2}{|c|}{ Non-transient erythema } \\
\hline Mild & $2(20)$ \\
\hline Moderate & $8(80)$ \\
\hline \multicolumn{2}{|c|}{ Papules and pustules } \\
\hline Absent & $4(40)$ \\
\hline Mild & $3(30)$ \\
\hline Moderate & $3(30)$ \\
\hline \multicolumn{2}{|l|}{ Telangiectasia } \\
\hline Moderate & $9(90)$ \\
\hline Severe & $1(10)$ \\
\hline \multicolumn{2}{|c|}{ Global assessment of rosacea } \\
\hline \multicolumn{2}{|c|}{ Subtype 1: erythematotelangiectatic } \\
\hline Mild & $1(10)$ \\
\hline Moderate & $8(80)$ \\
\hline Severe & $1(10)$ \\
\hline \multicolumn{2}{|c|}{ Subtype 2: papulopustular } \\
\hline Absent & $4(40)$ \\
\hline Mild & $3(30)$ \\
\hline Moderate & $3(30)$ \\
\hline \multicolumn{2}{|c|}{ Subtype 3: phymatous } \\
\hline Absent & $10(100)$ \\
\hline \multicolumn{2}{|c|}{ Subtype 4: ocular } \\
\hline Absent & $10(100)$ \\
\hline
\end{tabular}


Lanier et al.: Effects of a Facial Cream Containing the Minor Alkaloid Anatabine on Improving the Appearance of the Skin in Mild to Moderate Rosacea

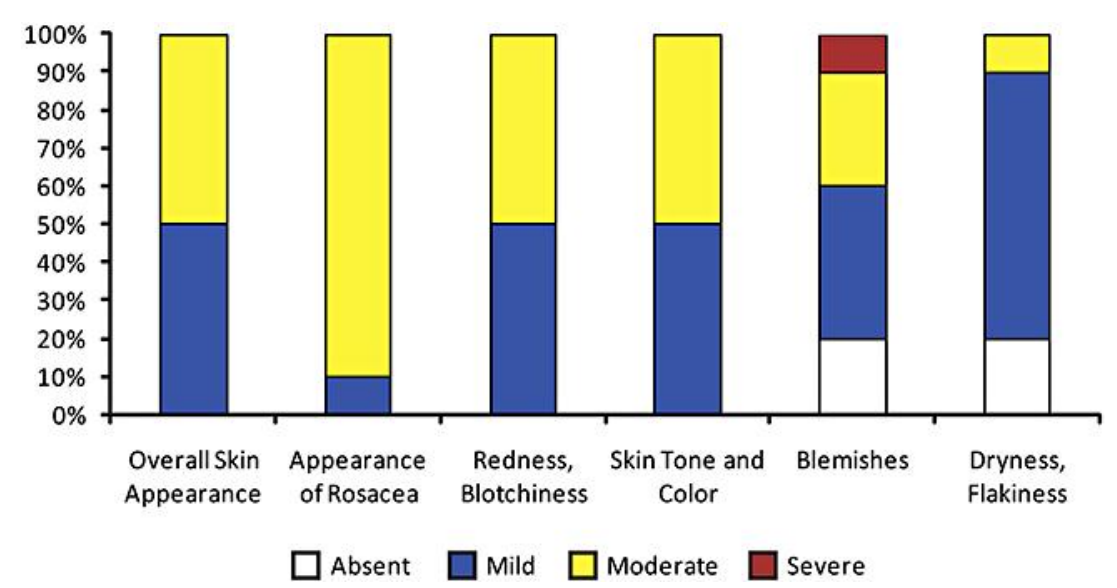

Fig. 1. PatGA of rosacea at baseline. Patients rated 6 components of their baseline skin appearance and rosacea symptoms (overall skin appearance; appearance of rosacea; redness/blotchiness; skin tone and color; blemishes, and dryness/flakiness) on a 4-point scale from 0 (absent) to +3 (severe) by filling out the PatGA. All study patients self-reported the overall appearance of rosacea to be either mild ( 1 of 10 , $10 \%$ ) or moderate ( 9 of $10,90 \%)$. Redness and blotchiness were reported as mild for $5(50 \%)$ of the patients and moderate for the remaining 5 (50\%) patients.

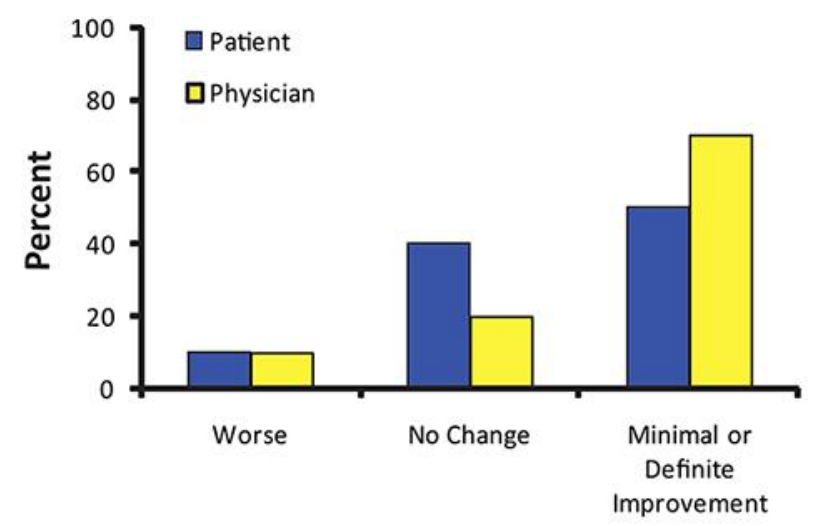

\section{Efficacy Rating}

Fig. 2. PatEA and PhyEA ratings. Patients and the physician rated the effectiveness of the cream for improving skin appearance after 30 days of twice daily use by filling out the PatEA and the PhyEA, respectively. The PatEA and PhyEA each consisted of a 5-point rating scale from -1 (worse) to +3 (cleared up). The physician rated rosacea improvement in 7 of the $10(70 \%)$ patients, and $5(50 \%)$ patients selfreported rosacea improvement. 


\section{Case Reports in Dermatology}

Lanier et al:: Effects of a Facial Cream Containing the Minor Alkaloid Anatabine on Improving the Appearance of the Skin in Mild to Moderate Rosacea
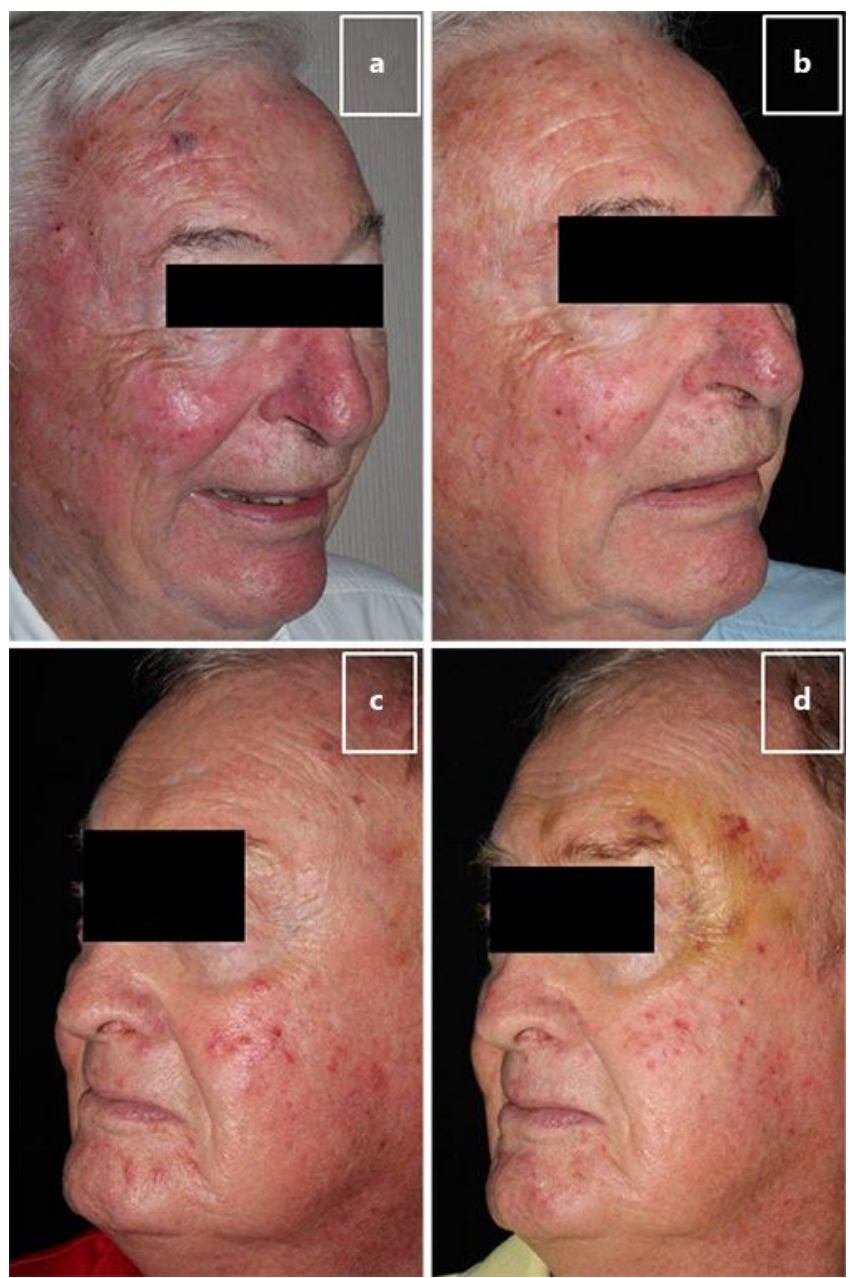

Fig. 3. Before and after photographs. Photographs showing 2 patients before (a, c) and after (b, d) 30 days of anatabine cream application. A marked reduction in facial erythema and improvement in the appearance of the skin was observed. 
Lanier et al.: Effects of a Facial Cream Containing the Minor Alkaloid Anatabine on Improving the Appearance of the Skin in Mild to Moderate Rosacea

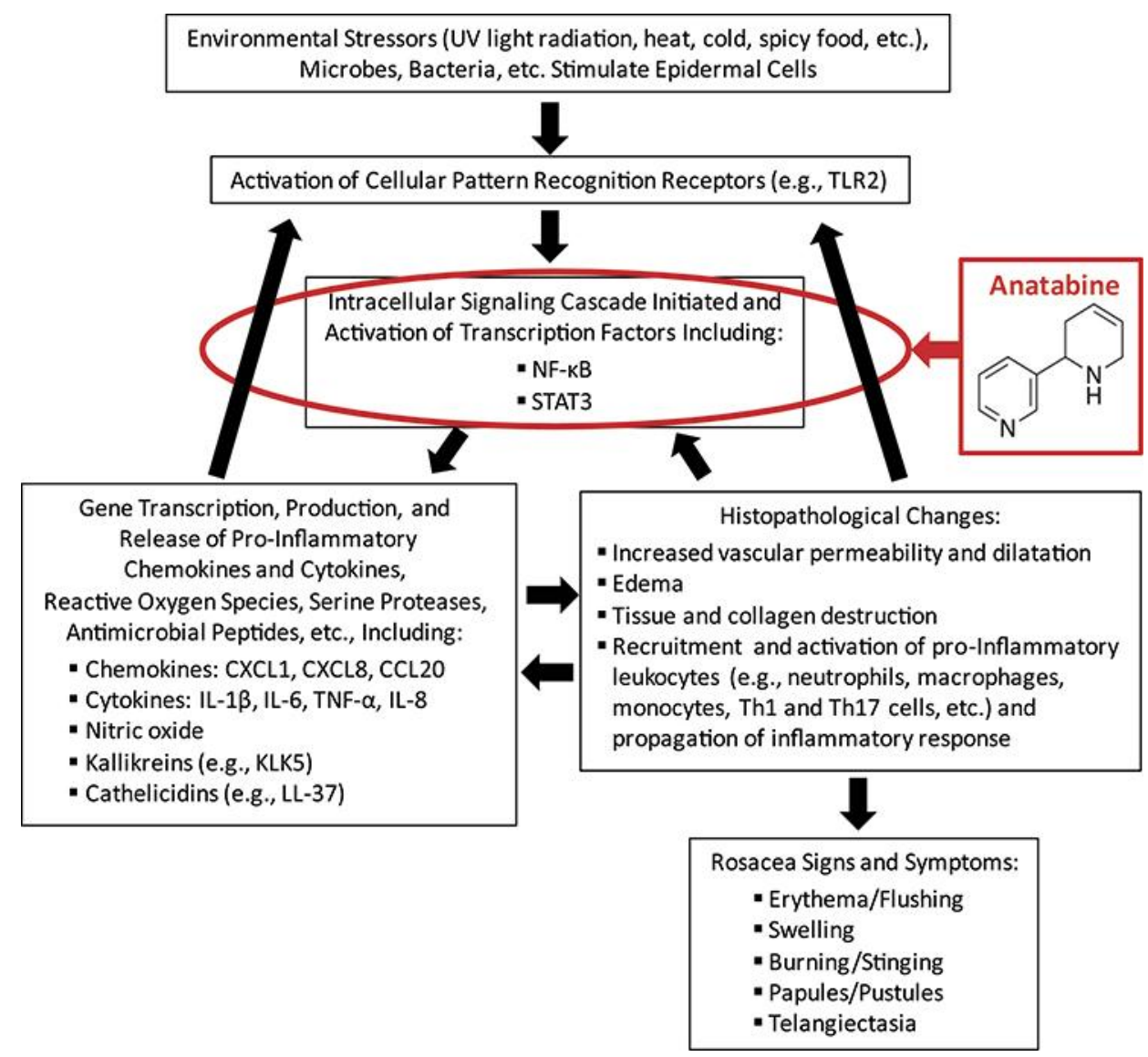

Fig. 4. Proposed mechanism of anatabine's effects on the proinflammatory signaling pathways involved in rosacea. The key molecular components believed to be involved in the proinflammatory signaling pathways and pathophysiology of rosacea are shown $[4-7,15]$. The proposed mechanism of anatabine's effects on rosacea signs and symptoms is through interference with the activation and translocation of transcription factors such as NF- $\mathrm{BB}$ and STAT3 involved in the propagation of proinflammatory signaling pathways. 